\title{
PROCESSAMENTO, CARACTERÍSTICAS E PROPRIEDADES DO NITRETO CÚBICO DE BORO - UMA REVISÃO ATUALIZADA*
}

Luiz Antonio Pessanha ${ }^{1}$ Michel Picanço

Ana Lucia Skury ${ }^{3}$

Sergio Neves Monteiro ${ }^{4}$ Luís Carlos da Silva ${ }^{5}$

\section{Resumo}

Apresenta-se uma revisão das características e propriedades do nitreto cúbico de boro (cBN) bem como de diferentes tipos de processamento. A influência da adição de ligantes e a formação de compósitos com outras cerâmicas são também revistas. Finalmente, destaca-se a grande importância prática do cBN como cerâmica de elevada dureza na substituição do diamante em ferramentas de corte.

Palavras-chave: Nitreto cúbico de boro, cBN, sinterização, dureza, aplicações.

\section{PROCESSING, CHARACTERISTICS AND PROPERTIES OF CUBIC BORON NITRIDE - A UPDATED REVIEW}

\begin{abstract}
A review of the characteristics and properties of cubic boron nitride (cBN) is presented, including different processing methods. The influence of bonding substances as well as other ceramic composites is also reviewed. Finally, it is highlighted the great practical importance of the $\mathrm{cBN}$ as a ceramic with elevated hardness in substitution for diamond in cutting tools.
\end{abstract}

Keywords: Cubic boron nitride, cBN, sintering, hardness, applications.

1 Engenheiro Metalúrgico, D.Sc., Doutor em Ciência dos Materiais, Seção de Engenharia Mecânica e de Materiais, Universidade Estadual Norte Fluminense, Rio de Janeiro, RJ, Brasil

2 Engenheiro Metalúrgico, D.Sc., Doutor em Ciência dos Materiais, Seção de Engenharia Mecânica e de Materiais, Universidade Estadual Norte Fluminense, Rio de Janeiro, RJ, Brasil

3 Engenheiro Metalúrgico, D.Sc., Doutor em Ciência dos Materiais, Seção de Engenharia Mecânica e de Materiais, Universidade Estadual Norte Fluminense, Rio de Janeiro, RJ, Brasil

4 Engenheiro Metalurgista, Ph.D, Professor Titular, Seção de Engenharia Mecânica e de Materiais, Instituto Militar de Engenharia, Rio de Janeiro, RJ, Brasil.

5 Engenheiro Químico, D.Sc., Doutor em Ciência dos Materiais, Seção de Engenharia Mecânica e de Materiais, Instituto Militar de Engenharia, Rio de Janeiro, RJ, Brasil. 


\section{INTRODUÇÃO}

O Nitreto cubico de boro (cBN) é uma das formas alotrópicas do nitreto de boro (BN), sintetizado sobre altas pressões e altas temperaturas (HPHT) [1]. O cBN é o segundo material mais duro, somente superado pelo diamante, além da alta dureza também é estável em altas temperaturas, possui baixa difusibilidade no Ferro $(\mathrm{Fe})$ e alta estabilidade química [3,4]. Segundo Solozhenko o cBN em baixa temperatura é um material estável, diferente do diamante [6]. Desde 1969 o cBN é um produto comercial, estando desde então o seu estudo em amplo crescimento [5]. O desenvolvimento e o estudo das técnicas de sinterização do cBN estão diretamente ligados ao avanço da indústria metal mecânica, assim como, da economia em geral [2].

A grande aplicação do $c B N$ é na forma policristalina ( $P c B N)$, utilizando principalmente na indústria metal mecânica, os primeiros relatos da sinterização do cBN formado PcBN foram realizados em 1963 em uma patente americana [7]. Desde então muitos estudos vem sendo desenvolvidos envolvendo as varias variáveis da sinterização do cBN. A sinterização do PcBN pode ser realizada utilizando altas pressões e altas temperaturas (HPHT), em pressões que variam de $2 \mathrm{GPa}$ até $20 \mathrm{GPa}$, e temperaturas que variam de $1300^{\circ} \mathrm{C}$ a $2500^{\circ} \mathrm{C}[3,5,6,7,8]$, podendo utilizar ligantes cerâmicos, metálicos ou sinterização direta.

Outra via de sinterização do cBN pode ser a sinterização em baixas pressões e altas temperaturas que tem por principal meio de sinterização o processo SPS (spark plasma sintering), utilizando pressões não superiores a $100 \mathrm{MPa}$ e temperaturas abaixo de $2200^{\circ} \mathrm{C}$ [4]. Na sinterização por SPS são utilizados ligantes cerâmicos e metálicos, assim como na sinterização em HPHT.

Tratando-se da sinterização do cBN é impossível abordar o tema sem antes fazer uma separação entre a magnitude das pressões utilizadas. Neste contexto serão estudados separadamente os processos que envolvem altas e baixas pressões.

\section{MATERIAIS E MÉTODOS}

Entre as variáveis do processo de sinterização ganham destaque a pressão, temperatura e a composição da mistura sinterizada. Neste contexto, a seguir serão abordados os trabalhos de sinterização do cBN utilizando altas pressões e altas temperaturas (HPHT).

$\mathrm{Na}$ tabela 1 são apresentados a composição do PcBN sinterizado sobre altas pressões. Os primeiros trabalhos tratando da sinterização do cBN tiveram início na década de 60, quase que em sua totalidade gerando patentes para Companhia General Eletric[ 1]. Os principais ligantes utilizados foram tungstênio, Níquel, berílio, rênio, titânio, zircônio, cromo, alumínio, cobalto, ferro, manganês e alumina e os elementos dos grupos IV-VI da tabela periódica $[1,12]$. 


\section{RESULTADOS E DISCUSSÃO}

Entre as variáveis do processo de sinterização ganham destaque a pressão, temperatura e a composição da mistura sinterizada. Neste contexto, a seguir serão abordados os trabalhos de sinterização do cBN utilizando altas pressões e altas temperaturas (HPHT).

$\mathrm{Na}$ tabela 1 são apresentados a composição do PcBN sinterizado sobre altas pressões.

\subsection{A importância do Al na sinterização do cBN.}

O principal ligante utilizado na sinterização do $\mathrm{cBN}$ é o $\mathrm{Al}$, a sua utilização ganhou destaque desde os primeiros trabalhos em que se estudava a sinterização do PcBN $[1,8,14]$.

Tabela 1: Composicão e parâmetros de sinterizacão do PcBN

\begin{tabular}{|c|c|c|c|c|c|c|c|c|c|c|c|c|c|c|}
\hline $\begin{array}{c}\text { Autor/ } \\
\text { referência }\end{array}$ & $\begin{array}{l}\text { \%cBN/ } \\
\text { (T.G.) }\end{array}$ & $\begin{array}{l}\text { \%hBN/ } \\
\text { (T.G) * }\end{array}$ & $\begin{array}{l}\% A L / \\
\text { (T.G) }\end{array}$ & $\begin{array}{l}\text { \%Co / } \\
\text { (T.G) }\end{array}$ & $\begin{array}{l}\text { \%V/I } \\
\text { (T.G) }\end{array}$ & $\begin{array}{l}\% \mathrm{Si} / \\
\text { (T.G) }\end{array}$ & $\begin{array}{l}\text { \%CTixN } \\
\text { y/ (T.G.) }\end{array}$ & $\begin{array}{l}\text { \%AIN/ } \\
\text { (T.G.) }\end{array}$ & $\begin{array}{l}\text { \% } \mathrm{TTiB}_{2} / \\
\text { (T.G.) }\end{array}$ & $\begin{array}{l}\text { \%TIN/ } \\
\text { (T.G.) }\end{array}$ & $\begin{array}{l}\text { \%TIC/ } \\
\text { (T.G.) }\end{array}$ & $\begin{array}{c}\text { \% } \mathrm{Ti}_{3} \mathrm{SiC}_{2} \\
\text { / (T.G) }\end{array}$ & $\begin{array}{c}\text { Pressão } \\
\text { (GPa) }\end{array}$ & $\begin{array}{c}\text { Temperatura } \\
\left({ }^{\circ} \mathrm{C}\right)\end{array}$ \\
\hline $\begin{array}{l}\text { BINDAL, } \\
1986 \text { [1] }\end{array}$ & $\begin{array}{c}40-60 \\
(6-9 \\
\mu \mathrm{m})\end{array}$ & & $\begin{array}{c}2-10(1 \\
\mu \mathrm{m})\end{array}$ & & & & $\begin{array}{c}45(2-5 \\
\mu \mathrm{m})\end{array}$ & & & & & & $5,5-6,2$ & $1300-1600$ \\
\hline $\begin{array}{c}\text { Hokamot } \\
\text { o,1997 } \\
{[11]}\end{array}$ & $\begin{array}{c}60-100 \\
(0-54 \\
\mu \mathrm{m})\end{array}$ & & & & & & & & $\begin{array}{l}0-40 \\
(1-2 \\
\mu \mathrm{m})\end{array}$ & & & & $11-26$ & $700-850$ \\
\hline
\end{tabular}

Tabela 2: Propriedades mecânicas dos PcBN segundo cada autor.

\begin{tabular}{|c|c|c|c|}
\hline Autor / referência & $\begin{array}{c}\text { Densificação } \\
(\%)\end{array}$ & Dureza & $\begin{array}{c}\text { Tenacidade } \\
\mathrm{MPa} \cdot \mathrm{m}^{-1 / 2}\end{array}$ \\
\hline BINDAL, 1986 [1] & --- & $2400-3600(\mathrm{GPa}) \mathrm{knM}$ & --- \\
\hline Hokamoto,1997 [11] & --- & $2-40(\text { GPA })_{\mathrm{HV}}$ & --- \\
\hline Bobrovnitchii, 2009 [10] & --- & $28-37(\mathrm{GPa}) \mathrm{KN}$ & $8,1-13,4$ \\
\hline Benko, 1999 [12] & --- & $16-28(\mathrm{GPa})_{\mathrm{HV}}$ & --- \\
\hline Benko, 1999 [12] & --- & 24-39 (GPa) $\mathrm{HV}$ & --- \\
\hline Sumiya, 2000 [13] & --- & 20-60 (GPa) HV & --- \\
\hline
\end{tabular}

Lv e colaboradores sinterizaram cBN-15wt.\%Al e cBN-20wt.\%AIN, utilizando pressões de $5 \mathrm{GPa}$ e temperaturas variando de 1300 a $1700^{\circ} \mathrm{C}$. Comparando os sistemas, melhores resultados de dureza foram alcançados para cBN-Al nas composições utilizadas, como está sendo mostrado no gráfico da figura 1[8]. 


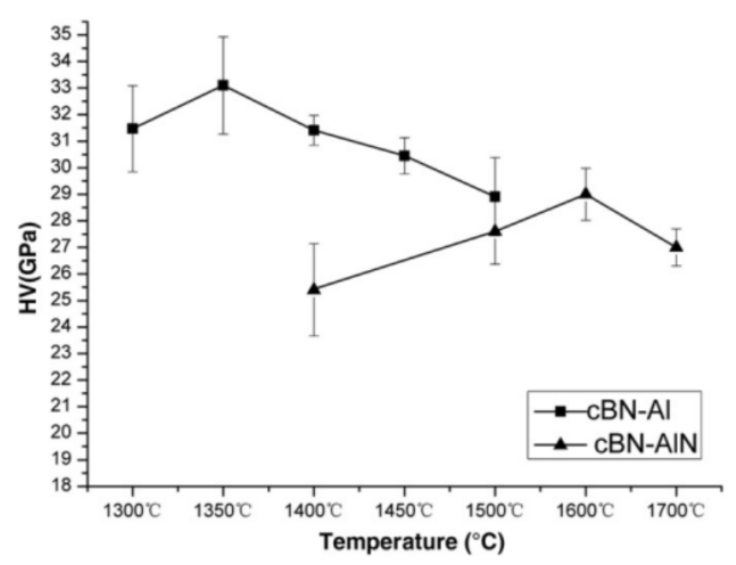

Figura 1: Comparação da dureza Vickers para os compactos PcBN sinterizados a partir de cBN-15wt.\% Al e cBN-20wt.\% AIN [8].

Atribui-se esses resultados a transformação do cBN-Al em AlB2 [8]. Tanto no trabalho de Lv e de Rong todo o Al foi consumido durante a sinterização, Lv propôs as seguintes transformações.

$\mathrm{Al}(\mathrm{I})+\mathrm{BN}(\mathrm{s})=\mathrm{AIN}(\mathrm{s})+\mathrm{B}^{*}$

$\mathrm{Al}(\mathrm{I})+2 \mathrm{~B}^{*}=\mathrm{AlB}_{2}(\mathrm{~s})$

Onde (*) significa átomos de $B$ dissolvidos no alumínio liquido [8]. Em que o aumento da temperatura favorece a formação de $\mathrm{AlB}_{2}$, e a sua formação diminui a dureza do PcBN $[8,14]$. Além disso, com o aumento da temperatura as transformações do cBN em hBN são ativadas. Para o sistema cBN-Al Wang e colaboradores encontraram em uma análise de DSC um pico proveniente dessa transformação em $1462^{\circ} \mathrm{C}$ [6].

A alta difusão do alumínio liquido, assim como, a reações entre o $\mathrm{Al}$ e o cBN são fatores que promovem uma alta sinterabilidade do sistema cBN-Al, promovendo um grande aumento de sua densificação, figura 2 [15]. Outra propriedade que tem um aumento significativo com o aumento da quantidade de Al é a condutividade térmica [15]. Essa propriedade ganha muita importância quando se trata de pastilhas de usinagem.

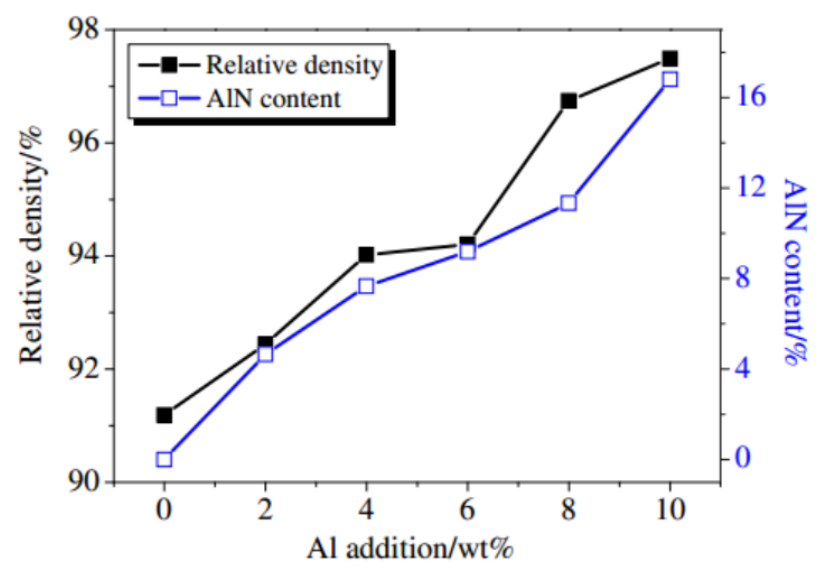

Figura 2: Variação da densidade relativa e porcentagem de AIN versus Al para sinterizados cerâmicos Al-CBN [15] 
Se com aumento da quantidade relativa de Al na sinterização as propriedades como condutividade e densificação tem uma melhora significativa, outras propriedades como dureza podem ter um decréscimo [9]. Foi o que demostrou McKie em seu trabalho, além de comparara os resultados de dureza com a quantidade de Al, também comparou com a granulometria do cBN. Com o aumento da granulometria do cBN McKie encontrou uma redução significativa da dureza, chegando em alguns casos quase a 50\%, para uma variação granulométrica de 2,5 a 12,5 $\mu \mathrm{m}$ [9].

Para os resultados de dureza, se comparado com a quantidade de ligante (Al) McKie, teve uma redução de aproximadamente $10 \%$, obtendo o valor máximo de $40 \pm 1,2$ $\mathrm{GPa}$ (hv) para uma variação de 20 a $40 \%$ em volume de, ligante [9]. Por outro lado, a tenacidade a fratura tende a aumentar com o aumento do tamanho de grão de cBN assim como, com o aumento do teor de Al [9]. Porém, para grãos em torno $17 \mu \mathrm{m}$, McKie encontrou uma relação inversa isto é, a tenacidade a fratura diminuiu com o aumento do teor de ligante.

Diferente de Wang, Rong e Lv no trabalho de McKie se encontrou alumínio residual, que não se transformou, por sua alta ductilidade esperava-se que esse alumínio contribuísse para o aumento da tenacidade a fratura. No entanto McKie concluiu que esse Al não afetou os resultados de tenacidade a fratura [9]. McKie atribuiu os autos valores de tenacidade a fratura ( 8MPa. $\left.\mathrm{m}^{1 / 2}\right)$ encontrados aos mecanismos de deflexão de trincas [9]. É importante ressaltar a presença das fases $\mathrm{AIN}_{\mathrm{N}}$ e $\mathrm{AlB}_{2}$, presentes na sinterização do $\mathrm{cBN}-\mathrm{Al}$, sempre aparecendo ao redor dos grãos de cBN $[1,8,8,14,16,21]$. A presença dessas fases e a ligação destas com o cBN são responsáveis pelos bons resultados de dureza e tenacidade a fratura encontrados nos PcBN-Al $[1,8,9,14,16,18]$.

Eko e colaboradores utilizaram $\mathrm{Co}, \mathrm{V}$ e Al na sinterização de $\mathrm{PcBN}$, em misturas de 10 e 20wt.\% de ligante no cBN [16]. O objetivo de Eko foi aumentar a dureza do PcBN, aumentando a presença de fases duras ao redor dos grãos de $c B N$, pois o $V N$ possui dureza maior que o AIN, (HV 25 GPa), (HV 11GPa) respectivamente [16]. Com a adição de $V$ e Co novas fases podem ser formadas como o nitreto de vanádio (VN) e uma solução solida de (Al-Co) em altas temperaturas segundo as equações 3 e 4 [16]. $2 \mathrm{~V}+\mathrm{N} 2=2 \mathrm{VN}$

$\mathrm{AlN}+\mathrm{V}+\mathrm{Co}=\mathrm{VN}+(\mathrm{Co}-\mathrm{Al})$

Eko acredita que devido a transformação do AIN em VN equação 4, a presença de AIN foi quase imperceptível nos difratogramas de DRX, resultados consistentes com os resultados de MEV, figura 3. Demonstrando que a presença de $V$ inibe a formação de AIN, dando lugar ao nitreto de vanádio, fase de maior dureza e almejada pelo autor [16]. 

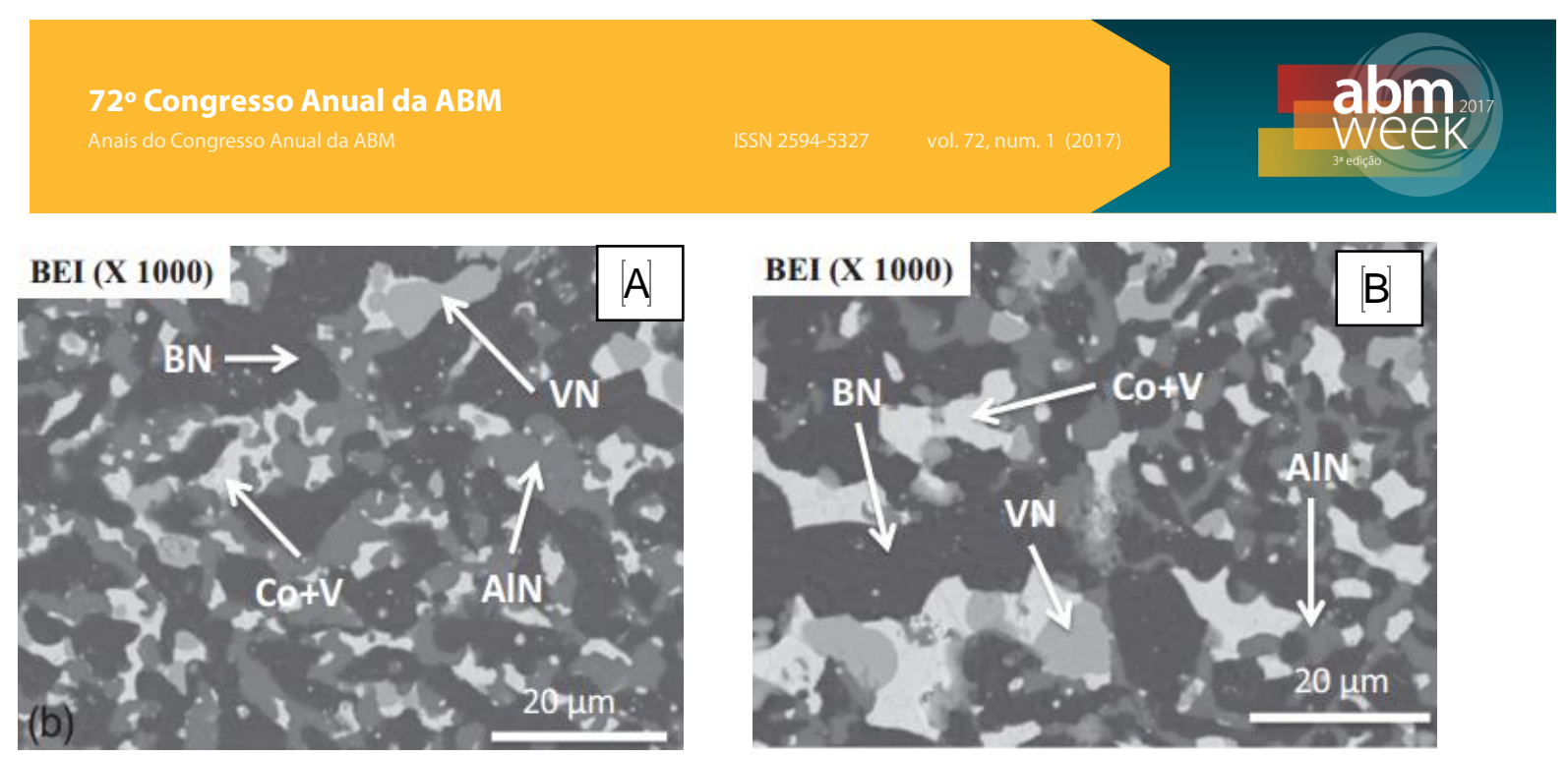

Figura 3: A- SEM-BEl imagens de hBN/Co65V25Al10 wt.\%/hBN amostra laminada sinterizada a $4.6 \mathrm{GPa}$ and $1400^{\circ} \mathrm{C}$ para $0.5 \mathrm{~h}$. B- SEM-BEl imagens de hBN/Co80V10Al10 wt.\%/hBN amostra laminada sinterizada a $5.6 \mathrm{GPa}$ e $1700{ }^{\circ} \mathrm{C}$ para $0.5 \mathrm{~h}[16]$.

Mesmo com a presença de NV substituindo o AIN os resultados de dureza alcançados por Eko [16] não obtiveram diferença significativa se comparado com os resultados de McKie [9], para quantidade de ligantes iguais (20wt.\%), e granulometria do cBN similar $(\sim 2 \mu \mathrm{m})$, em ambos os trabalhos.

Em uma outra tentativa de melhorar as propriedades do PcBN Zhang e colaboradores sinterizaram o cBN (70 wt.\% a 90 wt.\%) com Al e HfC 2:1 respectivamente [18]. A adição de $\mathrm{HfC}$ foi justificada pelo seu auto ponto de fusão, alta condutividade térmica e elétrica, além de, alta dureza (HV 33GPa), está muito superior a encontrada para AIN AIN (HV 11GPa) [16]. Através da análise das fases presentes Zhang encontrou outras fases que se transformaram durante a reação de sinterização, a equação 5 prevê a formação das fases acima de $1000^{\circ} \mathrm{C}$ [18].

$(2 \mathrm{x}+12) \mathrm{BN}+(3 \mathrm{x}+10) \mathrm{Al}+5 \mathrm{HfC}=(2 \mathrm{x}+10) \mathrm{AIN}+\mathrm{xAlB} 2+5 \mathrm{HfB}_{2}+\mathrm{B}_{2} \mathrm{C}_{5} \mathrm{~N}_{2}$

Segundo os resultados de DRX apresentados por Zhang ainda restou Al remanescente depois da sinterização, em seu artigo o autor não deixou claro se o alumínio formou solução solida com algum outro elemento [18]. Mesmo com a presença de $\mathrm{HfC}$ (fase de alta dureza), os resultados globais de dureza encontrados estão dentro do patamar dos resultados obtidos pelos outro autores ( 40GPa HV) [18]. Li e colaboradores sinterizaram (70-95wt.\%)cBN - Al sobre um substrato de WC16 wt. \%Co sob pressão de 5GPa e temperaturas variando de 800 a $1400^{\circ} \mathrm{C}$ [19]. Como já foi visto anteriormente todo Al reagiu durante a sinterização formando as fases AIN, $\mathrm{AlB}_{2}$, e solução solida com Co. A máxima dureza encontrada foi de 32,7GPa, abaixo do encontrado por outros autores. $O$ autor não deixou claro sobre a ligação entre a camada de cBN e o substrato. Porém, com as fases formadas $\mathrm{Al}-\mathrm{Co}_{\text {ss }}$ e $\mathrm{Al}_{3} \mathrm{~B}_{6} \mathrm{Co}_{20}$, pode-se acreditar que ocorreu uma boa ligação interfacial, visto que ocorreu difusão do Co para cBN-Al [19].

\subsection{Sinterização do cBN utilizando as cerâmicas a base de Ti (TiB2, TiC e TiN).}

Benko e colaboradores estudaram o equilíbrio químico, morfologia e resistência mecânica nos sistemas e cBN-TiC-TiN [12]. Estudando as variáveis termodinâmicas 
envolvidas no processo de sinterização em altas temperaturas (1000-1700ํㅡ), Benko determinou que em uma larga faixa de pressão o TiN reagirá com $c B N$ dando origem a $\mathrm{TiB}_{2}$.

Mesmo em sinterização em baixas pressões Benko conseguiu alcançar durezas significativas $40 \mathrm{GPa}$. Esses resultados podem ser atribuídos as fases cerâmicas de alta dureza utilizadas como ligante. A maior dureza alcançada é similar a dos resultados encontrados para os sistemas cBN-Al em HPHT [8,9,12,14,16,18,23]. Parte desses resultados pode ser explicado pela interação entre a o cBN e o ligante, como pode ser visto na microestrutura da figura 4 , não existe uma boa interação $c B N / c B N$

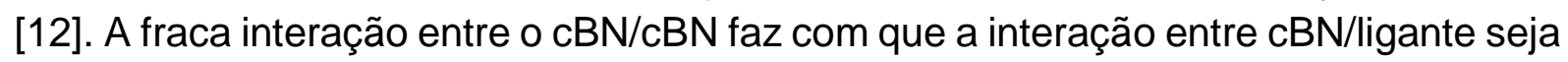
responsável pelas propriedades mecânicas do PcBN, no sistema cBN-TiN/TiC [12].

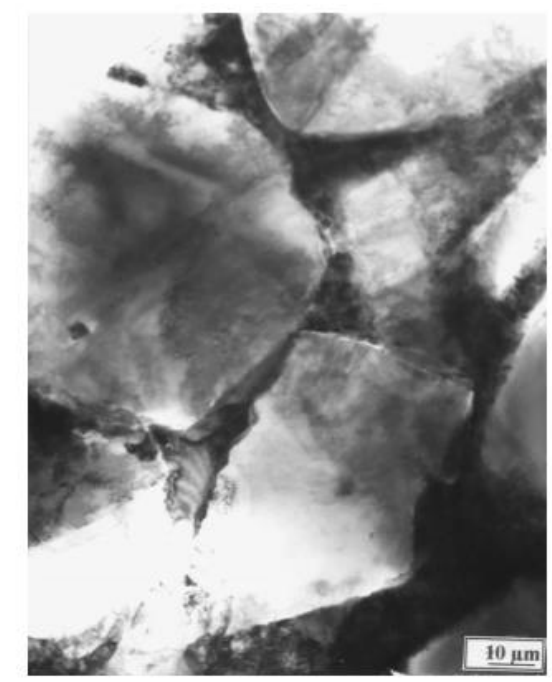

Figura 4: Microestrutura do compósito cBN-TiC

Sinterização em baixas pressões (SPS)

$\mathrm{O}$ cBN é conhecido pela sua baixa sinterabilidade, provocada por fortes ligações covalentes e baixo coeficiente de difusão entre o boro e o nitrogênio [23,24]. Neste sentido, a sinterização utilizando pressão ultra-alto (acima de 5GPa) e diferentes tipos de aditivos foi a primeira alternativa encontrada para obter elevada densificação de cBN. Uma vez que, pressão ultra-alta de sinterização é dispendiosa e empregada apenas para produtos de dimensões reduzidas, a utilização de um processo de sinterização com pressão moderada (inferior a $100 \mathrm{MPa}$ ) é vantajosa para a expansão da aplicação de cBN [20].

Spark plasma sintering (SPS) vem se tornando uma alternativa promissora para sinterização de materiais de difícil densificação. A SPS, mesmo utilizando pressão moderada, possibilita obter alta densificação utilizando temperaturas e tempo de sinterização inferior a técnica HPHT. Isto é possível através de uma eficaz ativação superficial e um efeito de auto-aquecimento entre as partículas, o que viabiliza a conclusão da transferência de calor e transferência de massa em praticamente de forma instantânea [20,22]. Além disso, alta taxa de aquecimento e curto tempo de permanência na temperatura máxima utilizada na técnica SPS restringe a indesejável transformação de cBN em hBN [23, 24]. 
Semelhante à técnica de sinterização por pressão a quente, a sinterização por plasma pulsado (SPS) também utiliza matrizes de grafite. No entanto, o aquecimento é obtido por descargas de faísca criadas nos vazios entre as partículas (figura 5). O SPS combina a aplicação simultaneamente de pressão e uma corrente elétrica contínua pulsada de alta intensidade e baixa tensão, fluindo diretamente através do pó, possibilitando taxas de aquecimento muito rápido [26, 27]. Diferentes tipos de materiais cerâmicos estruturais de alta densidade já foram fabricados com essa técnica, além de, cerâmicas transparentes e materiais intermetálicos [28].

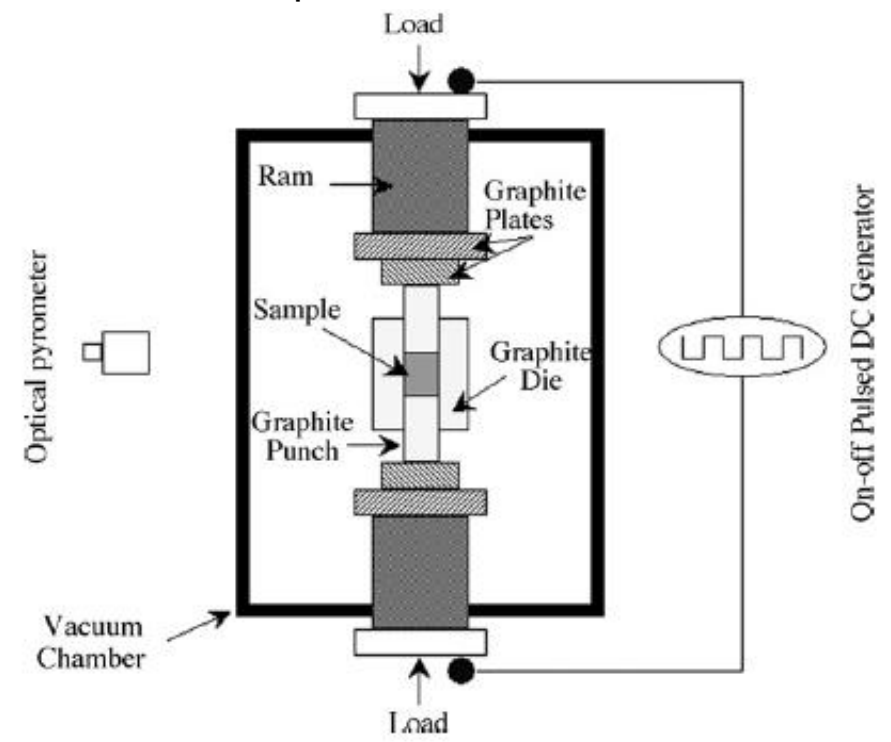

Figura 5: Representação esquemática do aparato por sinterização à plasma [28].

Mesmo o fato da Spark plasma sintering permitir uma transferência de calor e transferência de massa praticamente instantânea, e consequentemente, densificação total em curto espaço de tempo e sinterização do cBN exige a utilização de aditivos. P. Klimczyk et al [36] mostrou que Spark sinterização plasma pode ser considerada uma alternativa promissora ao dispendioso processo HPHT na preparação de compósitos a base de cBN. Os autores utilizaram ambos os métodos para avaliar a sinterização do nitreto de boro cúbico (cBN) utilizando como ligante $30 \%$ em volume de $\mathrm{Al}_{2} \mathrm{O}_{3}$. Após otimização da temperatura de sinterização, tanto para HPHT e o método SPS, para atingir a maior densidade e módulo de Young possível das amostras, os autores observaram que amostras obtidas por HPHT apresentaram melhores propriedades físicas e elásticas quando comparadas as amostras sinterizadas por SPS.

\section{CONCLUSÃO}

Desde sua sintetização em 1969, o nitreto cúbico de boro (cBN) vem apresentando uma aumento em suas aplicações como um dos principais substitutos do diamante em ferramentas para a indústria metal-mecânica.

O alumínio tem sido o principal ligante na sinterização do cBN, garantindo alto grau de sinterabilidade bem como aumento na condutividade térmica. 
Compósitos contendo cBN em matriz de cobalto apresentam boa dureza com elevada tenacidade à fratura, o que favorece a sua utilização como pastilhas de corte.

\section{REFERÊNCIAS}

1. M. M. BINDAL, R. K. NAYAR, S. K. SINGHAL, A JAY DHAR, RAJEEV CHOPRA, High-pressure sintering of cubic boron nitride, JOURNAL OF MATERIALS SCIENCE 21 (1986) 4347-4351

2. J. C. WALMSLEY, A. R. LANG, A transmission electron microscope study of a cubic boron nitride-based compact material with AIN and AIB2 binder phases. JOURNAL OF MATERIALS SCIENCE 22 (1987) 4093-4102.

3. H. Sumiya $\square$, K. Harano, Y. Ishida, Mechanical properties of nano-polycrystalline cBN synthesized by direct conversion sintering under HPHT, Diamond \& Related Materials 41 (2014) 14-19.

4. $\quad$ Yungang Yuan, Xiaozhe Cheng, Rui Chang, Tianheng Li, Jianbing Zang, Yanhui Wang, Yiqing Yu, Jing Lu, Xipeng Xu, Reactive sintering cBN-Ti-Al composites by spark plasma sintering, Diamond \& Related Materials 69 (2016) 138-143.

5. Laurence Vel, Grard Demazeau and Jean Etourneau, Cubic boron nitride: synthesis, physicochemical properties and applications, Materials Science and Engineering, B IO ( 1991$)$ 149-164.

6. V. L. Solozhenko, Dokl. Akad. Nauk S.S.S.R., 301 (1) (1988) 147.

7. Process for making polycrystalline cubic boron nitride and product therefrom. US Patent 3078232, 1963.

8. Ran Lv, Jin Liu, Yongjun Li, Sicheng Li, Zili Kou, Duanwei He, High pressure sintering of cubic boron nitride compacts with Al and AIN, Diamond \& Related Materials 17 (2008) 2062-2066.

9. Amanda McKie, Jami Winzer, lakovos Sigalas, Mathias Herrmann, Ludwig Weiler, Jurgen Ro, Nedret Can, Mechanical properties of cBN-Al composite materials, Ceramics International 37 (2011) 1-8.

10. Guerold Bobrovnitchii, Ana Lúcia Diegues Skury, Marcello Filgueira, Rômulo Crespo Tardim, PASTILHAS DE cBN PARA APLICAÇÃO EM USINAGEM DE AÇOS

TEMPERADOS, V CONGRESSO BRASILEIRO DE ENGENHARIA DE FABRICAÇÃO, abril de 2009 - Belo Horizonte - Minas Gerais - Brasil.

11. K. Hokamoto, S. Tanaka, M. Fujita, S. Itoh, M.A. Meyers, H.-C. Chen, High temperature shock consolidation of hard ceramic powders, Physica B 239 (1997) I-5. 12. Ewa Benkoa, Jan Skrzypek Stanisławb, Bogna Kro lickaa, A. Wyczesanyc, Tery L. Barr, cBN-TiN, cBN-TiC composites: chemical equilibria, microstructure and hardness mechanical investigations, Diamond and Related Materials 8 ( 1999) 1838-1846. 13. H. SUMIYA, S. UESAKA, S. SATOH, Mechanical properties of high purity polycrystalline $\mathrm{cBN}$ synthesized by direct conversion sintering method, JOURNAL OF MATERIALS SCIENCE35(2000)1181 - 1186.

14. Xiao-Zheng Rong, Takaaki Tsurumi, Osamu Fukunaga, Toyohiko Yano, Highpressure sintering of $\mathrm{cBN}$-TiN-Al composite for cutting tool application, Diamond and Related Materials 11(2002)280-286.

15. P.F. Wang, Zh. H. Li, Y.M. Zhu, Fabrication of high thermal conductive AlecBN ceramic sinters by high temperature high pressure method, Solid State Sciences 13 (2011) $1041 \mathrm{e} 1046$.

16. Akhmadi Eko, Osamu Fukunaga, Naoto Ohtake, High pressure sintering of cubic boron nitride using Co-V-Al alloy as bonding media, Int. Journal of Refractory Metals and Hard Materials 50 (2015) 178-183.

17. Lili Zhang, FengLin, ZhiLv, ChaoXu, Xulin He,WenlongWang, Liwei Li, Changlong Zhang, ChaoChen, Luojun Xia, cBN-Al-HfC composites: Sintering behaviors and mechanical properties under high pressure, Int. Journal of Refractory Metals and Hard Materials 50 (2015) 221-226. 
18. V.L. Solozhenko, V.Z. Turkevich, W.B. Holzapfel, Refined phase diagram of boron nitride, Journal of Physical Chemistry B 103 (15) (1999) 2903-2905.

19. F.P. Bundy, R.H. Wentorf Jr., Direct transformation of hexagonal boron nitride to denser forms, Journal of Chemical Physics 38 (5) (1963) 1144- 1149.

20. H. Moriguchi, K. Tsuduki, A. Ikegaya, When diamonds and CBN are a driller's best friends, Metal Powder Report 59 (4) (2004) 26-30.

21. Z. Shen, M. Johnsson, Z. Zhao, M. Nygren, Spark plasma sintering of alumina, J. Am. Ceram. Soc. 85 (2002) 1921-1927.

22. J. Song, K. Ma, Y. Li, L. Zhang, J.M. Schoenung, High temperature microstructure and microhardness evolution in dense NiCrAIY bulk material fabricated by spark plasma sintering, Mater. Sci. Eng. A 528 (2011) 3210-3217.

23. K. Brookes, Making hardmetal even harderwith dispersed cBN,Met. Powder Rep. 62 (2007) 14-17.

24. B. Yaman, H. Mandal, Spark plasma sintering of Co-WC cubic boron nitride composites, Mater. Lett. 63 (2009) 1041-1043.

25. Orru R, Licheri R, Locci AM, Cincotti A, Cao G. Consolidation/synthesis ofmaterials by electric current activated/assisted sintering. Mater Sci Eng 2009;R 63:127-287.

26. Hungria T, Galy J, Castro A. Spark plasma sintering as a useful technique to the nanostructuration of piezo-ferroelectric materials. Adv Eng Mater 2009;11(8):615-31.

27. Y. Zhao, et al., Preparation and microstructure of a ZrB2-SiC composite fabricated by the spark plasma sintering-reactive synthesis (SPS-RS) method, Journal of the American Ceramic Society 90 (12) (2007) 4040-4042.

28. I. Akin, et al., Microstructure and densification of ZrB2-SiC composites prepared by spark plasma sintering, Journal of the European Ceramic Society 29 (11) (2009) 2379-2385. 